\title{
Neuroprotective and MAOB inhibitory effects of a series of caffeine-8-thioglycolic acid amides
}

\author{
Alexandra Kasabova-Angelova ${ }^{1 *}$, Magdalena Kondeva-Burdina ${ }^{\circledR 1}$, Javor Mitkov², \\ Maya Georgieva ${ }^{2}$, Virginia Tzankova ${ }^{1}$, Alexander Zlatkov ${ }^{2}$

\begin{abstract}
${ }^{1}$ Laboratory "Drug Metabolism and Drug Toxicity", Department "Pharmacology, Pharmacotherapy and Toxicology", Faculty of Pharmacy, Medical University-Sofia, Sofia, Bulgaria, ${ }^{2}$ Department "Pharmaceutical chemistry", Faculty of Pharmacy, Medical University-Sofia, Sofia, Bulgaria
\end{abstract}

\begin{abstract}
The effects of new derivatives of caffeine-8-thioglycolic acid $(100 \mu \mathrm{M})$ on isolated rat brain synaptosomes, human neuroblastoma cell line $\mathrm{SH}-\mathrm{SY} 5 \mathrm{Y}$ and human recombinant MAOB enzyme (hMAOB) $(1 \mu \mathrm{M})$ were evaluated. Most of the compounds, administered alone, didn't show statistically significant neurotoxic effects on $\mathrm{SH}-\mathrm{SY} 5 \mathrm{Y}$, when compared to the control (non-treated cells). Of all studied structures JTA-2Ox, JTA-11, JTA-12 and JTA-13 decreased cell viability. In combination with 6-hydroxydopamine (6-OHDA) $(100 \mu \mathrm{M})$, only JTA-1 and JTA-2 revealed neuroprotective effects, stronger than those of caffeine. All compounds administered alone revealed, neurotoxic effects on synaptosomes, as compared to nontreated synaptosomes. JTA-1, JTA-2 and JTA-3 showed lowest neurotoxic effects and were investigated in a model of 6-OHDA-induced oxidative stress. In this model of neurotoxicity, only JTA-1 and JTA-2 showed statistically significant neuroprotective effect, by preserving the synaptosomal viability and the level of reduced glutathione. Inhibition of hMAOB, was revealed by JTA-1 and JTA-2. They inhibited the enzyme by $23 \%$ and $25 \%$ respectively, thus approaching the selegiline activity, which was $42 \%$. The possible mechanisms of neuroprotection of JTA-1 and JTA-2 might be a result from the inhibition of hMAOB, which catalyze the production of neurotoxic p-quinone from 6-OHDA.
\end{abstract}

Keywords: Neuroprotection. Caffeine. Synaptosomes. SH-SY5Y. hMAOB.

\section{INTRODUCTION}

Neurodegenerative diseases are characterized by loss of neurons in certain regions of the brain. They are incurable and debilitating conditions that result in progressive degeneration and/or death of nerve cells. This causes problems with movement (ataxias), or mental functioning (dementias).

For a couple of decades, significant ageing of the population has been observed worldwide and the prevalence of such disorders is growing continuously. At present, therapeutic strategies for treatment of neurodegenerative diseases are only symptomatic and none of them is able to terminate or delay the disease progression.

\footnotetext{
*Correspondence: A. Kasabova-Angelova. Laboratory "Drug Metabolism and Drug Toxicity", Department "Pharmacology, Pharmacotherapy and Toxicology", Faculty of Pharmacy, Medical University-Sofia, 2 Dunav Str., Sofia, Bulgaria. Tel.: +359 29236 573. Fax.: +359 2987 9874. E-mail: akasabovaangelova@gmail.com
}

Parkinson's disease (PD) is one of the most common chronic neurodegenerative diseases following Alzheimer's disease (Tieu, 2011). Approximately 630,000 people in US were diagnosed with PD only in 2010 (Kowal et al., 2013). The symptoms of the disease were described by James Parkinson in his monograph "An Essay on the Shaking Palsy" for the first time in 1817. Parkinsonism is a syndrome characterized by resting trepidation, rigidity, delayed or absent conscious movements and postural instability. So far, there has no cure been found and the causes of the disease remain not fully understood, with less than $10 \%$ of the cases being directly associated with monogenic mutations (Tieu, 2011).

$\mathrm{PD}$ is a progressive disease caused by damage of the central nervous system nigrostriatal neuronal pathways. The pathophysiological distinguishing features of PD are loss of dopaminergic neurons in substantia nigra pars compacta and the presence of cytoplasmic protein aggregates called Lewy bodies. When degeneration of these neurons reaches a threshold of about $60 \%$ and 
striatal dopamine decreases by about $80 \%$, symptoms of PD appear (Dauer, Przedborski, 2003).

The current treatment of PD is generally focused in compensating dopamine deficiency, which is ineffective in delaying progression. Dopamine replacement therapy is associated with many side effects, especially dyskinesia. Recent progress in understanding of molecular mechanisms involved in $\mathrm{PD}$ has revealed new perspectives for PD therapy. In these strategies, caffeine and xanthine derivatives are promising scaffolds for design of novel structures with valuable neuroprotective properties.

Although pathogenesis of PD remains unclear, there is an increasing evidence for the involvement of mitochondrial function, oxidative damage and inflammation in the development of the neurodegenerative process (Dauer, Przedborski, 2003; Bagga, Patel, 2016)

Caffeine (1,3,7-trimethylxanthine) is a natural alkaloid and similarily toxanthines, act as non-selective adenosine $\mathrm{A}_{2 \mathrm{~A}}$ receptor antagonist. Caffeine easily crosses the blood-brain barrier and exhibits a variety of behavioral effects, including sharpened alertness, reduced fatigue and increased motor activity (Smith, 2002) by inhibiting the suppression of neuronal function (Garrett, Griffiths, 1997).

Several clinical trials and meta-analyzes have showed that caffeine consumption is associated with a decreased risk of developing PD and dementia (Ross, Petrovitch, 2001; Ascherio et al., 2001; Costa et al., 2010; Altman, Lang, Postuma, 2011; Palacios et al., 2012; Liu et al., 2012; Kolahdouzan, Hamadeh, 2017).

Other studies report similar results and the evidence for the potential beneficial effects of caffeine and xanthine derivatives administration is growing.

An investigation on the effect of pre-treatment with caffeine on the expression of genes in MPTPlesioned mouse striatum, has been performed by Singh and colleagues in 2010. Several genes responsible for apoptotic cell death, oxidative stress, cell cycle regulation, protein modification and mitochondrial dysfunction were examined. The results revealed significant reduction of damaging events after caffeine treatment (Singh et al., 2010).

Sonsalla and co-workers found out in their study that caffeine administration upon the onset or throughout the course of ongoing neurodegeneration, reduced the loss of dopaminergic neurons in substantia nigra and might stop or slow down the degeneration process (Sonsalla et al., 2012).

In their paper, Kaster and co-workers (2015) revealed the relation of caffeine and istradefylline with mood and memory alterations provoked by chronic stress. The results show that chronic stress induces behavioral and synaptic alterations and xanthine based structures, such as caffeine and istradefylline, prevented those alterations (Kaster et al., 2015).

A lot of efforts have been made to uncover the therapeutic potential of xanthine structures as neuroprotective agents over the last decades. The substituted xanthine molecule is used as a scaffold for the synthesis of new compounds with protective effects in neurodegenerative diseases. The use of the xanthines has been proposed as a non-dopaminergic strategy for neuroprotection in Parkinson's disease and the mechanisms of protection have been associated with antagonism of adenosine $\mathrm{A}_{2 \mathrm{~A}}$ receptors and monoamine oxidase type $\mathrm{B}$ (MAOB) inhibition (Palacios et al., 2012). Adenosine $\mathrm{A}_{2 \mathrm{~A}}$ receptor antagonism leads to reduced inflammation through decreased proliferation and activity of the astroglial cells in the midbrain (Bagga, Patel, 2016; Madeira et al., 2017). On the other hand, the neuroprotective effects of MAOB inhibitors can be explained in part, taking into account the metabolic products generated by the action of MAOB on monoamines. In the catalytic reaction of MAOB, aldehyde product and hydrogen peroxide are produced for each mole of oxidized monoamine substrate (Petzer et al., 2009). These metabolic products may be neurotoxic, if they are not rapidly inactivated by antioxidant systems, whether enzymatic or non-enzymatic.

Additionally, some data indicate a possible dual target activity of methylxanthine derivatives with pronounced neuroprotective effect, which is expressed in a potent and selective antagonism on adenosine $\mathrm{A}_{2 \mathrm{~A}}$ receptors coupled with $\mathrm{MAOB}$ inhibition (Petzer et al., 2009).

Based on this data, a series of synthesized caffeine-8-thioglycolic acid amides was evaluated for possible neuroprotective effects as MAOB inhibitors. The neurotoxicity of the compounds on $S H-S Y 5 Y$ neuroblastoma cells was also investigated.

\section{MATERIAL AND METHODS}

\section{Synthesis of caffeine-8-thioglycolic acid (KTG) derivatives (JTA1 to JTA 13)}

A modified method of Persch and Beyerle (1957) was used to synthesize caffeine-8-thioglycolic acid (Persch, Beyerle, 1957). The reaction was carried out in $60 \%$ ethanol and the yield was $92 \%$. Methyl caffeine-8-thioglycolate was prepared by the method used for synthesizing ethyl esters of 7-alkylxanthinyl8 -thioglycolic acids. The reaction was carried out in anhydrous methanol in the presence of a catalytic amount 
of sulfuric or phosphoric acid and the yield was $95 \%$. After aminolysis of methyl caffeine-8-thioglycolate, a series of amide derivatives were obtained (Mitkov et al., 2007). The structures of the synthesized amide derivatives were proven by IR, ${ }^{1} \mathrm{H}$ and ${ }^{13} \mathrm{C}$-NMR spectral data. The purity of the compounds was confirmed by the corresponding TLC characteristics, melting points and elemental analyses.

\section{Chemicals}

The chemicals used in the experiments:

- 3-(4,5-dimethylthiazol-2-yl)-2,5-diphenyltetrazolium bromide (MTT), Roswell Park Memorial Institute (RPMI) 1640 Medium, Fetal Bovine Serum 10\%, L-glutamine, 6-OHDA, Percoll, 4-(2-hydroxyethyl)-1-piperazineethanesulfonic acid (HEPES), sucrose, 2-thiobarbituric acid (4,6-dihydroxypyrimidine-2-thiol) and Tyramine hydrochloride were obtained from Sigma Aldrich, Germany;

- $\mathrm{NaCl}, \mathrm{KCl}, \mathrm{CaCl}_{2} \cdot 2 \mathrm{H}_{2} \mathrm{O}, \mathrm{MgCl}_{2}, \mathrm{NaHPO}_{4}, \mathrm{D}-$ glucose, trichloroacetic acid, 2,2'-dinitro-5,5'dithiodibenzoic acid (DTNB) and selegiline were obtained from Merck, Germany;

Amplex UltrRed Kit was obtained from Invitrogen, USA.

\section{Cultivation and incubation of human neuroblastoma cell line $\mathrm{SH}-\mathrm{SY} 5 \mathrm{Y}$}

The $S H-S Y 5 Y$ human neuroblastoma cell line was received from Sigma Aldrich (ECACC cell lines). The cultivation process was carried out with nutritional media RPMI-1640, supplemented with fetal bovine serum 10\% and L-glutamine. Sub-cultivation process was carried twice a week. When about $80 \%$ confluence was reached the cells were seeded in 96-well plate, and placed for $24 \mathrm{~h}$ in an incubator under standard conditions $\left(37^{\circ} \mathrm{C}\right.$, $5 \% \mathrm{CO}_{2}$ ) for precipitation and adhesion (seeding cell density $3.10^{4}$ per well). The cells were incubated with solutions of the test substances in nutrient medium (final concentration $100 \mu \mathrm{M}$ ) (Timson, 1970) followed by 24 hours incubation.

\section{Model of neurotoxicity on SH-SY5Y cell line}

The neuroblastoma cells were incubated with $100 \mu \mathrm{M} 6-O H D A$ and the test substance for 24 hours. In our preliminary experiments, we investigated 5 different concentrations of the toxic agent: $50 \mu \mathrm{M}, 100 \mu \mathrm{M}$, $150 \mu \mathrm{M}, 200 \mu \mathrm{M}$ and $250 \mu \mathrm{M}$ and as a result we found that concentration $100 \mu \mathrm{M}$ of 6 -OHDA lead to $50 \%$ cell death and was convenient for the experiments.

\section{MTT test: Vitality assessment method}

The tetrazole salt, MTT, is reduced by the action of mitochondrial dehydrogenases to water insoluble violet crystals formazan. The culture medium was aspirated and added $100 \mu \mathrm{L}$ of a solution of freshly prepared MTT $(0.5 \mathrm{mg} / \mathrm{mL})$ in culture medium and 3 hours incubation was carried out. The liquid from the wells was aspirated and added $100 \mu \mathrm{L}$ of dimethyl sulfoxide (DMSO) and maintained 10 minutes with shaking at dark. Absorbance was measured at $570 \mathrm{~nm}$ and $690 \mathrm{~nm}$. The amount of formed formazan is proportional to viability (Mosmann, 1983).

\section{Animals}

Male Wistar rats (body weight 200-250 g) were used. The rats were housed in plexiglass cages ( 3 per cage) in a 12/12 light/dark cycle, under standard laboratory conditions (ambient temperature $20 \pm 2{ }^{\circ} \mathrm{C}$ and humidity $72 \pm 4 \%$ ) with free access to water and standard pelleted rat food 53-3, produced according ISO 9001:2008. The animals were purchased from the National Breeding Center, Sofia, Bulgaria. Seven days acclimatization was allowed before the commencement of the study and a veterinary physician monitored the health of the animals regularly. Vivarium (certificate of registration of farm No. 0072/01.08.2007) was inspected by the Bulgarian Drug Agency in order to check the husbandry conditions (No. A-11-1081/03.11.2011). All performed procedures were approved by the Institutional Animal Care Committee and the principles stated in the European Convention for the Protection of Vertebrate Animals used for Experimental and other Scientific Purposes (ETS 123) (Council of Europe, 1991) were strictly followed throughout the experiment.

\section{Isolation and incubation of synaptosomes}

The synaptosomes were prepeared by multiple, subcellular fractionation using a Percoll gradient (Taupin et al., 1994). Two types of buffers were prepared - Buffer A: HEPES $5 \mathrm{mM}$ and Sucrose 0,32 M; Buffer B: $\mathrm{NaCl} 290 \mathrm{mM}, \mathrm{MgCl}_{2} .2 \mathrm{H}_{2} \mathrm{O} 0,95 \mathrm{mM}, \mathrm{KCl} 10 \mathrm{mM}$, $\mathrm{CaCl}_{2} \cdot 2 \mathrm{H}_{2} \mathrm{O} 2,4 \mathrm{mM}, \mathrm{NaH}_{2} \mathrm{PO}_{4} 2,1 \mathrm{mM}$, HEPES $44 \mathrm{mM}$, D-Glucose $13 \mathrm{mM}$.

Brain homogenates were prepared with buffer A and centrifuged at $1000 \mathrm{x}$ g for 10 minutes at $4{ }^{\circ} \mathrm{C}$. After centrifugation, the supernatants were removed and centrifuged again under the above-mentioned conditions. Supernatants were taken and subjected to three times centrifugation at $10000 \mathrm{x}$ g for 20 minutes at $4{ }^{\circ} \mathrm{C}$. The last two centrifuges were for purification of the synaptosomes. 
Isolation of synaptosomes was performed with the help of a colloidal silicon solution (Percoll).

1. Preparation of $90 \%$ stock solution of Percoll.

2. Preparation of Percoll solutions with two concentrations $16 \%$ and $10 \% .4 \mathrm{~mL}$ of Percoll $16 \%$ and $10 \%$, were added in the tubes.

3. $90 \%$ Percoll (7.5\% Percoll) was added to the sediment from the last centrifugation The tubes were centrifuged for 20 minutes at $15000 \mathrm{x}$ g at $4{ }^{\circ} \mathrm{C}$.

After centrifugation, three layers were formed in the tubes. The lower layer contained mitochondria, the upper layer - lipids and the middle layer (between $16 \%$ and $10 \%$ Percoll) - synaptosomes. The middle layer of each tube was harvested and buffer B with glucose was added. The mixture was centrifuged at $10000 \mathrm{x}$ g for 20 minutes at $4{ }^{\circ} \mathrm{C}$. After centrifugation, the sediment with the synaptosomes was mixed with buffer B with glucose.

The synaptosomes and the cells were incubated with $100 \mu \mathrm{M}$ of the test substances (Timson, 1970).

After the incubation, MTT-test was performed to determine synaptosomal vitality by method described by Mungarro-Menchaca et al. (2002).

\section{Determination of reduced glutathione (GSH)}

The level of reduced glutathione was determined by measuring the non-protein $\mathrm{SH}$-groups after precipitation of the proteins with trichloroacetic acid.

After the incubation, synaptosomes were centrifuged at $400 \mathrm{x}$ g for 3 minutes. The sediment was treated with $5 \%$ trichloroacetic acid and left for 10 minutes on ice. Samples were centrifuged at $8000 \mathrm{x}$ g for 10 minutes $\left(2{ }^{\circ} \mathrm{C}\right)$. The supernatant was removed to determine the level of GSH and was stored at $-20^{\circ} \mathrm{C}$. Immediately before the measurement, the samples were neutralized with $5 \mathrm{~N}$ $\mathrm{NaOH}$.

The presence of thiols in the supernatant was determined using Elmman reagent. The resulting yellow color was measured spectrophotometrically $(\lambda=412 \mathrm{~nm})$ (Robyt, Ackerman, Chittenden, 1971).

\section{Model of 6-OHDA-induced neurotoxicity}

This in vitro model resembles the neurodegenerative processes occurring in PD. Dopamine metabolism and oxidation lead to the formation of reactive oxygen species (ROS) and reactive quinones. They induce dopamine neurotoxicity and neurodegeneration (Stokes et al., 2002). The synaptosomes were incubated with $150 \mu \mathrm{M} 6$-OHDA and the test substance for 1 hour. After centrifugation, on a microfuge, the probes samples were centrifuged for 1 minute at $15000 \mathrm{x}$ g. The pellet was mixed with Buffer $\mathrm{B}$ and centrifuged again for 1 minute at $15000 \mathrm{xg}$. The precipitate containing the synaptosomal fraction was mixed with buffer B with glucose.

\section{Measurement of Monoamine oxidase B activity}

Monoamine oxidase activity assay of recombinant human MAOB was performed using a fluorometric method by Amplex UltraRed reagent (Bautista-Aguilera et al., 2014) with small modifications. Tyramine hydrochloride was used as substrate.

\section{Statistical methods}

Statistical analysis was performed using statistical programme "MEDCALC". Results are expressed as mean \pm SEM for 6 experiments. The significance of the data was assessed using the non-parametric MannWhitney test (synaptosomes). Values of $p \leq 0,05 ; p \leq 0,01$ and $\mathrm{p} \leq 0,001$ were considered statistically significant. The cell survival data were normalized as percentage of the control (set as $100 \%$ viability). Statistical evaluation was performed using the GraphPad Prism 5.0 software.

\section{RESULTS AND DISCUSSION}

The general procedure for the preparation of the desired amides is described elsewhere (Mitkov et al., 2007) and shown in Figure 1.

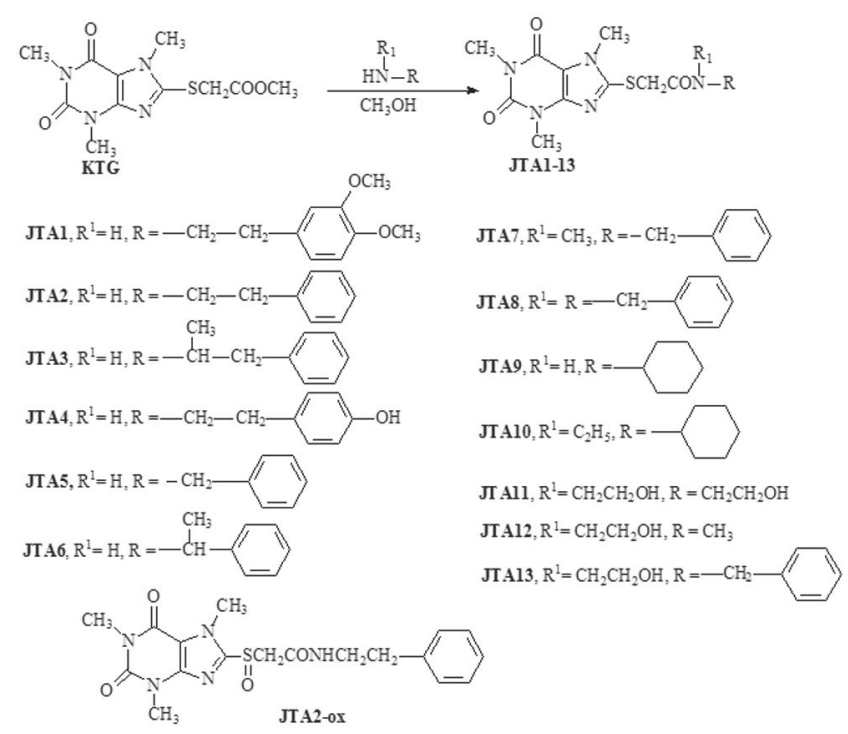

FIGURE 1 - Synthesis of caffeine-8-thioglycolic acid (KTG) amides. 
Effects of newly synthesized caffeine-8-thioglycolic acid (KTG) derivatives on neuroblastoma cell line SH-SY5Y

The $S H-S Y 5 Y$ neuroblastoma cells are often used as in vitro models of neuronal function and differentiation. They are adrenergic in phenotype but also express dopaminergic markers and therefore are appropriate to study Parkinson's disease.

Caffeine and caffeine-8-thioglycolic acid (KTG), administered alone, didn't reveal statistically significant neurotoxic effects, compared to the control (non-treated cells). Only JTA-2Ox, JTA-11, JTA-12 and JTA-13 decreased significantly the cell viability by $41 \%, 25 \%$, $37 \%$ and 39\%, respectively (Figure 2).

Some data suggest the presence of free radical scavenging activity of caffeine and other methylxanthines (Shi, Dalal, Jain, 1991). Thus, it was of interest to determine the effect of the less toxic caffeine amides JTA-1 - JTA-10 on a model of 6-OHDA-induced oxidative stress in SH-SY5Y cell line.

When administered alone, 6-OHDA decreased the cell viability with $48 \%$, compared to the control (nontreated cells). After the administration of the evaluated structures on this model, only JTA-1 and JTA-2 revealed good statistically significant neuroprotective effects, better than those of caffeine-8-thioglycolic acid (KTG) and caffeine. JTA-1 preserved cell viability by $56 \%$ and JTA- 2 by $54 \%$, while caffeine- 8 -thioglycolic acid and caffeine - by $31 \%$, compared to the toxic agent (Figure 3).

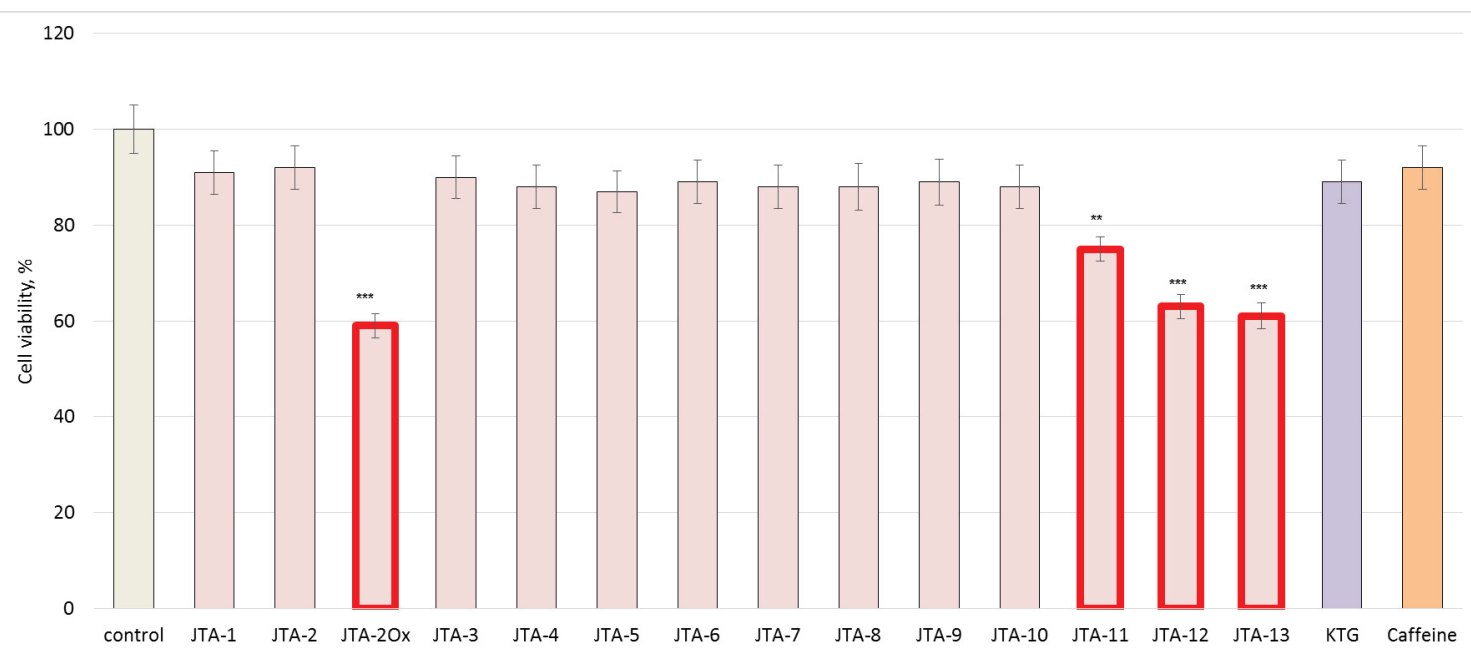

FIGURE 2 - Effects of caffeine-8-thioglycolic acid (KTG) derivatives, administered alone, on SH-SY5Y cell viability, measured by MTT-test. After $24 \mathrm{~h}$ of treatment with test substances cell viability is expressed as a percentage of a control (non-treated cells). Mean $\pm \operatorname{SEM}(\mathrm{n}=6) . * * \mathrm{P}<0.01 ; * * * \mathrm{P}<0.001-$ vs control.

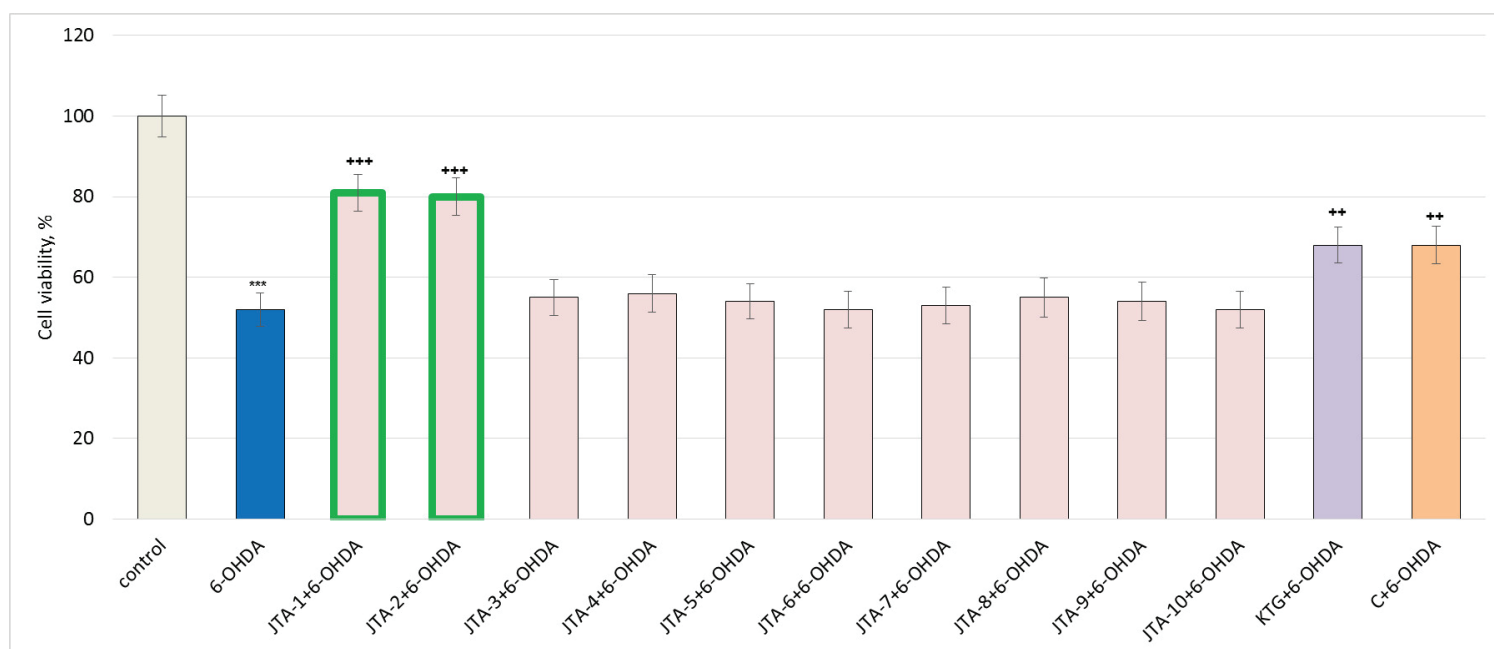

FIGURE 3 - Effects of JTA-1 to JTA-10, in conditions of 6-OHDA-induced oxidative stress, on SH-SY5Y cell viability, measured by MTT-test. After $24 \mathrm{~h}$ of treatment with test substances and toxic agent, cell viability is expressed as a percentage of a control (non-treated cells). Mean $\pm \operatorname{SEM}(\mathrm{n}=6) .{ }^{* * *} \mathrm{P}<0.001-$ vs control; ${ }^{++} \mathrm{P}<0.01 ;{ }^{++} \mathrm{P}<0.001-$ vs 6-OHDA. 
Effects of newly synthesized caffeine-8-thioglycolic acid (KTG) derivatives on isolated rat brain synaptosomes

Along with the other brain models (brain slices, primary neuronal cultures), the isolated nerve terminals - synaptosomes, are an important tool for investigation of the synaptic function in the brain on molecular level (Evans, 2015).

Using this useful model system for assessment of the neuroprotective properties of the studied amides we evaluated their effect on isolated rat brain synaptosomes.

The necessary rat brain synaptosomes were prepared by using Percoll reagent. All the tested derivatives, along with the initial caffeine and KTG expressed statistically significant neurotoxic effects on synaptosomal viability and level of GSH, compared to the control (non-treated synaptosomes).

Only compounds JTA-1, JTA-2 and JTA-3 presented lower neurotoxic effects on isolated rat brain synaptosomes (Figures 4 and 5). These 3 compounds were further evaluated for possible neuroprotective effects on a model of 6-OHDA-induced oxidative stress.

Based on data showing that caffeine prevents lipid peroxidation (Devasagayam et al., 1996) we determined the effect of the less toxic amide derivatives on 6-OHDA induced oxidative stress.

The treatment of isolated rat brain synaptosomes with 6-OHDA is a reliable and commonly used in vitro model for the investigation of processes, which play role in the neurodegenerative disease, including Parkinson's and Alzheimer's disease. The mechanism of 6-OHDA neurotoxicity includes the formation of ROS and reactive metabolites, as a result of its metabolism in mitochondria of the neuronal cells (Stokes et al., 2002).

The mechanism of destruction of the nerve terminals is thought involvement of oxidation of 6-OHDA to a $p$-quinone and production of a free radical or of superoxide anion. The species intermediate reacts covalently with the nerve terminal and permanently inactivates it (Timbrell, 2003).

When administered alone, 6-OHDA decreased synaptosomal viability and GSH level by $55 \%$ and $50 \%$, respectively, compared to the non-treated synaptosomes, while both, JTA-1 and JTA-2 revealed stronger than JTA-3 statistically significant neuroprotective effects compared to 6-OHDA by preserving the synaptosomal viability and level of GSH to higher extent (Figure 6).

JTA-1 preserved synaptosomal viability and level of GSH by $56 \%$ and $44 \%$, respectively, compared to toxic agent, while JTA-2 preserved synaptosomal viability and level of GSH by $58 \%$ and $46 \%$, respectively, compared to toxic agent. The neuroprotective effects of JTA- 1 and JTA-2 were similar to those of caffeine and KTG on this model of oxidative stress.

The third non-toxic amide JTA-3 - preserved the synaptosomal viability and level of GSH by $24 \%$ and $20 \%$, respectively, compared to the toxic agent, which demonstrates its lower effect than those of caffeine and KTG.

Effects of 14 newly synthesized derivatives of caffeine-8-thioglycolic acid (KTG) and selegiline on the activity of $h M A O B$ enzyme

MAOB is involved in the neurodegenerative process associated with aging, and is known to participate in neurodegenerative diseases including Parkinson's and Alzheimer's disease. MAOB inhibitors could prevent dopaminergic neuron degeneration (Timbrell, 2003) and reduce parkinsonian symptoms (Löhle, Storch, 2012). MAOB inhibitors could decrease metabolism of dopamine

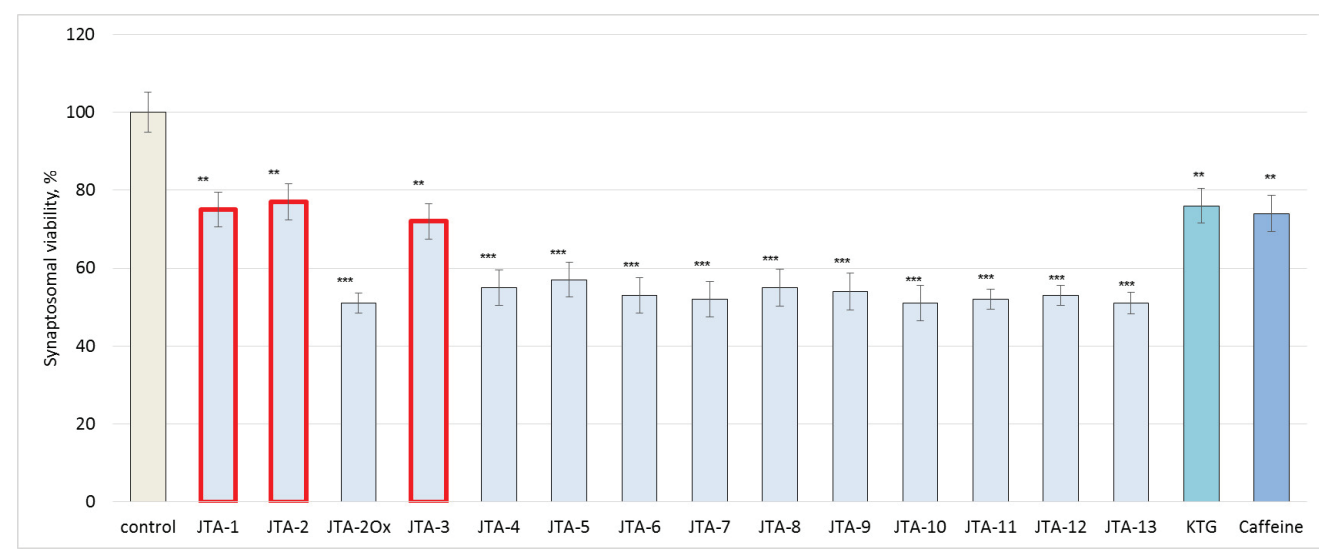

FIGURE 4 - Effects of caffeine-8-thioglycolic acid (KTG) derivatives, administered alone, on synaptosomal viability, measured by MTT-test. After $1 \mathrm{~h}$ of treatment with test substances synaptosomal viability is expressed as a percentage of a control (non-treated synaptosomes). Mean $\pm \operatorname{SEM}(\mathrm{n}=6)$. ${ }^{* *} \mathrm{P}<0.01 ; * * * \mathrm{P}<0.001-$ vs control. 


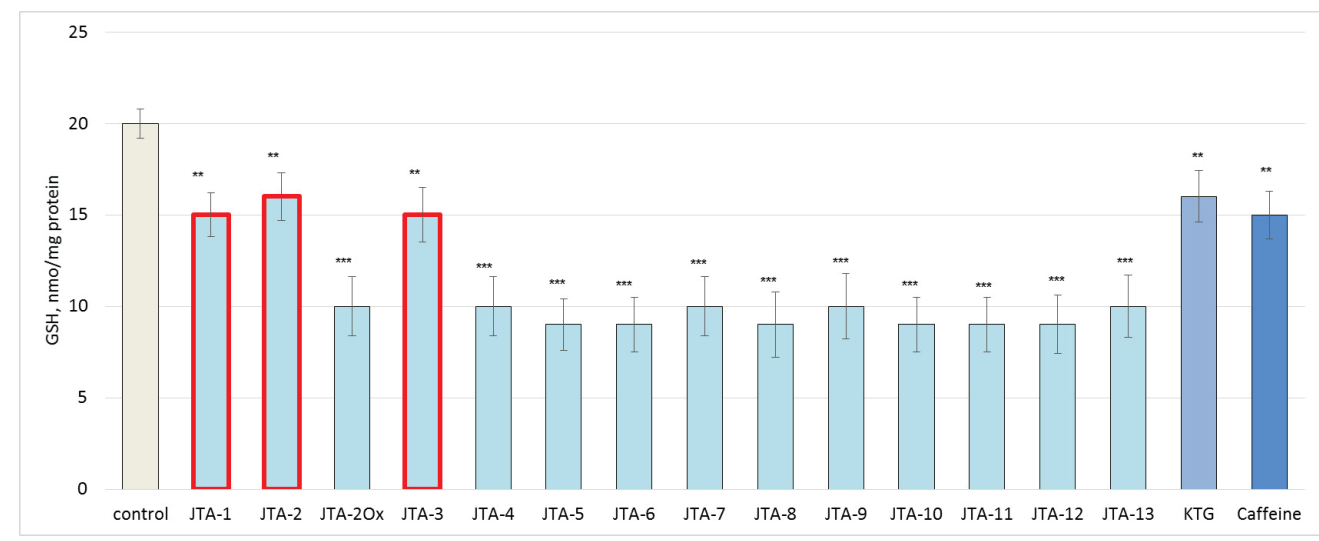

FIGURE 5 - Effects of caffeine-8-thioglycolic acid (KTG) derivatives, administered alone, on level of GSH after $1 \mathrm{~h}$ of treatment. Mean $\pm \operatorname{SEM}(\mathrm{n}=6) . * * \mathrm{P}<0.01 ; * * * \mathrm{P}<0.001-\mathrm{vs}$ control (non-treated synaptosomes).

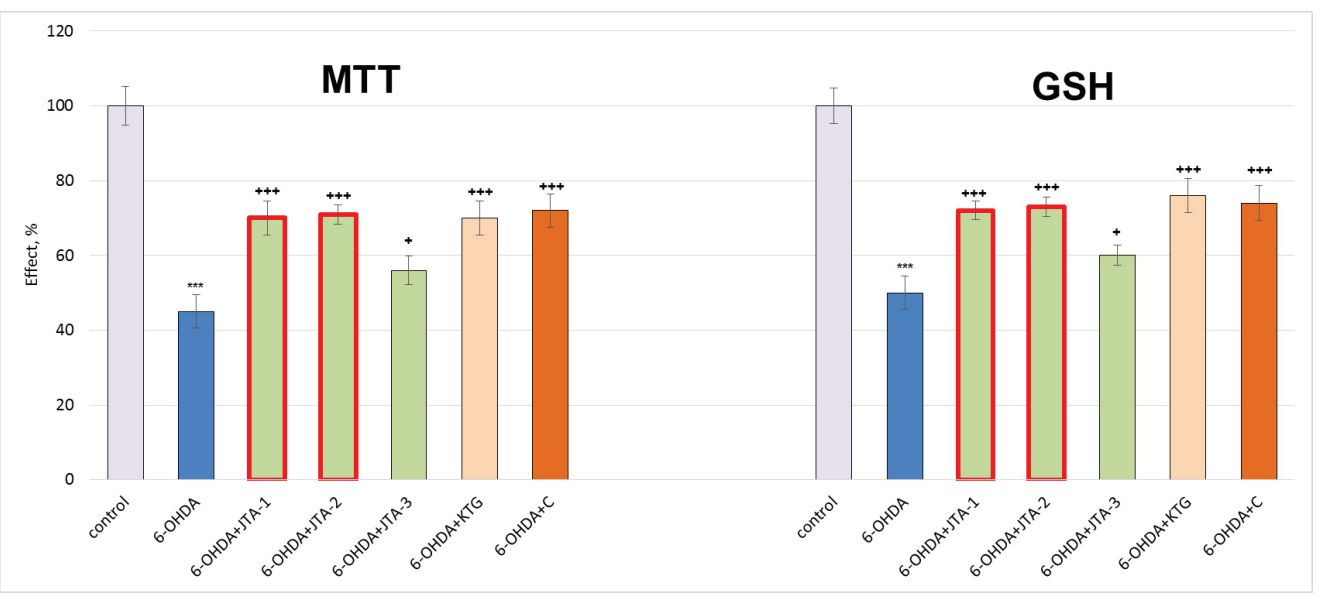

FIGURE 6 - Effects of JTA-1, JTA-2 and JTA-3 on synaptosomal viability and level of GSH in conditions of 6-OHDA-induced oxidative stress, after $1 \mathrm{~h}$ treatment. Mean $\pm \mathrm{SEM}(\mathrm{n}=6) .{ }^{* * *} \mathrm{P}<0.001-$ vs control (non-treated synaptosomes); ${ }^{+} \mathrm{P}<0.05 ;{ }^{+++}$ $\mathrm{P}<0.001-$ vs toxic agent (6-OHDA).

selectively and balance its concentration in synaptic cleft in a relatively stable state (Timbrell, 2003).

Based on the information on dual-target-acting agents developed in the last years as neuroprotective molecules, we assessed newly synthesized caffeine-8thioglycolic acid amides JTA-1-JTA-13 for potential MAOB inhibitory activity.

The results revealed that most of the examined compounds administered alone (at concentration $1 \mu \mathrm{M}$ ) didn't have statistically significant inhibitory effects on the activity of hMAOB, if compared to the control (pure hMAOB).

Only two of the compounds: JTA-1 and JTA-2 showed significantly good inhibitory activity on hMAOB, closer to Selegiline's activity. JTA-1 inhibited hMAOB by $23 \%$ and JTA- 2 - by $25 \%$, compared to pure hMAOB (Figure 7).

Selegiline (at concentration $1 \mu \mathrm{M}$ ) inhibited hMAOB by $42 \%$.
This study, lead us to the following conclusions:

In $S H-S Y 5 Y$ cell line, most of the derivatives, administered alone, didn't show statistically significant neurotoxic effects, compared to the control (non-treated cells). In conditions of 6-OHDAinduced oxidative stress, only JTA-1 and JTA-2 revealed good neuroprotection which was better than that exerted by caffeine-8-thioglycolic acid and caffeine.

On rat brain synaptosomes, administered alone, all compounds revealed statistically significant neurotoxic effects compared to the control (non-treated synaptosomes). JTA-1, JTA-2 and JTA-3 showed lowest neurotoxic effects and were investigated in a model of 6-OHDA-induced oxidative stress. Under the conditions of 6-OHDA neurotoxicity, only JTA-1 and JTA-2, with proven antihypoxic effects, showed significant neuroprotective effects on brain synaptosomes. 


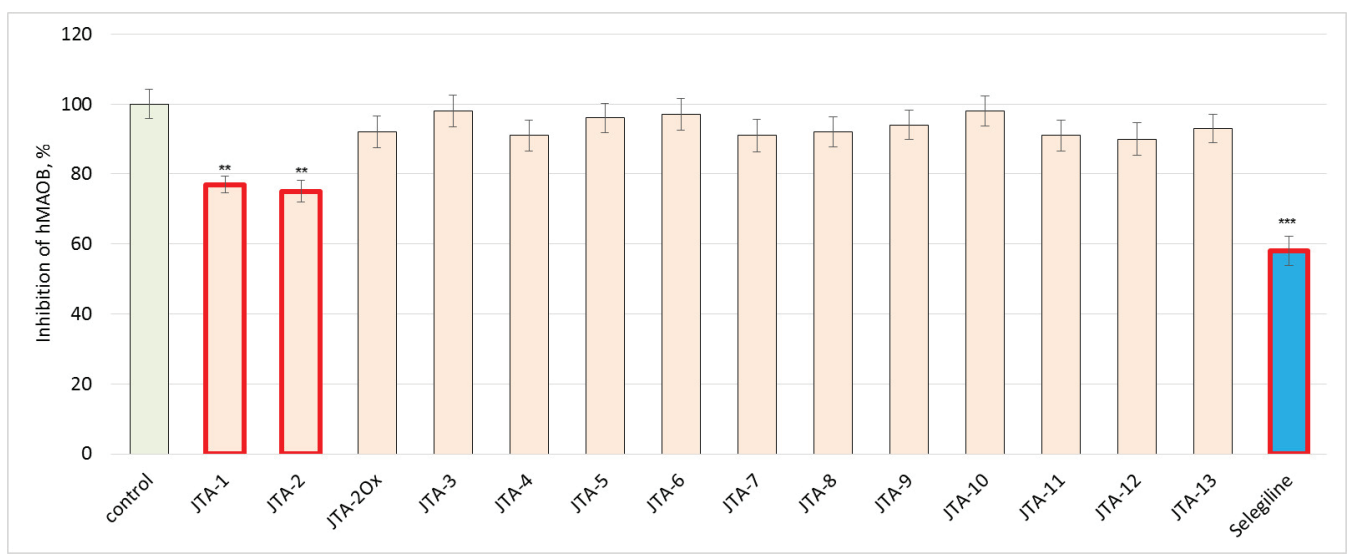

FIGURE 7 - Effects of caffeine-8-thioglycolic acid (KTG) derivatives and selegiline (at concentration $1 \mu \mathrm{M}$ ), on hMAOB enzyme. Mean $\pm \operatorname{SEM}(\mathrm{n}=6) . * * \mathrm{P}<0.01 ; * * * \mathrm{P}<0.001$ vs control (pure hMAOB).

- $\quad$ Statistically significant inhibitory activity on hMAOB, was revealed by JTA-1 and JTA-2. They inhibited the enzyme by $23 \%$ and $25 \%$, respectively, which was close to selegiline's effect, which inhibited hMAOB by $42 \%$.

- $\quad$ The possible mechanisms of JTA-1 and JTA-2 neuroprotection on 6-OHDA-induced toxicity model might be due to the inhibition of hMAOB, which catalyze the production of neurotoxic p-quinone from 6-OHDA.

- $\quad$ The obtained results highlight JTA-1 and JTA2 as the most promising compounds for further consideration as a structural base for design of new neuroprotective agents.

\section{ACKNOWLEDGEMENTS}

This study was support and financed by Contract №52/27.05.2016, Project №570/21.01.2016 of Counsel of Medical Sciences, Medical University-Sofia.

\section{REFERENCES}

Altman RD, Lang AE, Postuma RB. Caffeine in Parkinson's disease: a pilot open-label, dose-escalation study. Mov Disord. 2011;26(13):2427-31.

Ascherio A, Zhang SM, Hernan MA, Kawachi I, Colditz GA, Speizer FE, et al. Prospective study of caffeine consumption and risk of Parkinson's disease in men and women. Ann Neurol. 2001;50(1):56-63.

Bagga P, Patel AB. Pretreatment of caffeine leads to partial neuroprotection in MPTP model of Parkinson's disease. Neural Regen Res. 2016;11(11):1750-1751.
Bautista-Aguilera OM, Esteban G, Bolea I, Nikolic K, Agbaba D, Moraleda I, et al. Design, synthesis, pharmacological evaluation, QSAR analysis, molecular modeling and ADMET of novel donepezil-indolyl hybrids as multipotent cholinesterase/ monoamine oxidase inhibitors for the potential treatment of Alzheimer's disease. Eur J Med Chem. 2014;75:82-95.

Costa J, Lunet N, Santos C, Santos J, Vaz-Carneiro A. Caffeine exposure and the risk of Parkinson's disease: a systematic review and meta-analysis of observational studies. J Alzheimers Dis. 2010;20(Suppl 1):S221-38.

Dauer W, Przedborski S. Parkinson's disease: mechanisms and models. Neuron. 2003; 39(6):889-909.

Devasagayam TP, Kamat JP, Mohan H, Kesavan PC. Caffeine as an antioxidant: inhibition of lipid peroxidation induced by reactive oxygen species. Biochim Biophys Acta. 1996;1282(1):63-70.

Evans GJ. The synaptosome as a model system for studying synaptic physiology. Cold Spring Harb Protoc. 2015;2015(5):421-4.

Garrett BE, Griffiths RR. The role of dopamine in the behavioral effects of caffeine in animals and humans. Pharmacol Biochem Behav. 1997;57(3):533-41.

Kaster MP, Machado NJ, Silva HB, Nunes A, Ardais AP, Santana M, Baqi Y, Müller CE, Rodrigues AL, Porciúncula LO, Chen JF, Tomé ÂR, Agostinho P, Canas PM, Cunha RA. Caffeine acts through neuronal adenosine $\mathrm{A}_{2 \mathrm{~A}}$ receptors to prevent mood and memory dysfunction triggered by chronic stress. Proc Natl Acad Sci USA. 2015;112(25):7833-8. 
Kolahdouzan M, Hamadeh MJ. The neuroprotective effects of caffeine in neurodegenerative diseases. CNS Neurosci Ther. 2017;23(4):272-290.

Kowal SL, Dall TM, Chakrabarti R, Storm MV, Jain A. The current and projected economic burden of Parkinson's disease in the United States. Mov Disord. 2013;28(3):311-8.

Liu R, Guo X, Park Y, Huang X, Sinha R, Freedman ND, et al. Caffeine intake, smoking, and risk of Parkinson disease in men and women. Am J Epidemiol. 2012;175(11):1200-7.

Löhle M, Storch A. Effects of monoamine oxidase Type B inhibitors on motor and non-motor symptoms in Parkinson's disease: A systematic comparison of rasagiline and selegiline. Basal Ganglia. 2012;2(4 Suppl):S33-S40.

Madeira MH, Boia R, Ambrósio AF, Santiago AR. Having a coffee break: the impact of caffeine consumption on microgliamediated inflammation in neurodegenerative diseases. Mediators Inflamm. 2017;2017:4761081.

Mitkov J, Danchev N, Nikolova I, Zlatkov A. Synthesis and brain antihypoxic activity of some aliphatic and arylaliphatic amides of caffeine-8-thioglycolic acid. Acta Pharm. 2007;57(3):361-70.

Mosmann T. Rapid colorimetric assay for cellular growth and survival: application to proliferation and cytotoxicity assays. J Immunol Method. 1983;65(1-2):55-63.

Mungarro-Menchaca X, Ferrera P, Moran J, Arias C. Betaamyloid peptide induces ultrastructural changes in synaptosomes and potentiates mitochondrial dysfunction in the presence of ryanodine. J Neurosci Res. 2002;68(1):89-96.

Palacios N, Gao X, McCullough ML, Schwarzschild MA, Shah R, Gapstur S, et al. Caffeine and risk of Parkinson's disease in a large cohort of men and women. Mov Disord. 2012;27(10):1276-82.

Persch W, Beyerle R. Mercury-containing xanthine compounds. Ger Pat Chem. Abstr, 1957;53:18070d.

Petzer JP, Castagnoli NJ, Schwarzschild MA, Chen JF, Van der Schyf CJ. Dual-target-directed drugs that block monoamine oxidase B and adenosine A(2A) receptors for Parkinson's disease. Neurotherapeutics. 2009;6(1):141-51.
Robyt JF, Ackerman RJ, Chittenden CG. Reaction of protein disulfide groups with Ellman's reagent: a case study of the number of sulfhydryl and disulfide groups in Aspergillus Oryzae - Amylase, Papain and Lysosome. Arch Biochem Biophys. 1971;147:262.

Ross GW, Petrovitch H. Current evidence for neuroprotective effects of nicotine and caffeine against Parkinson's disease. Drugs Aging. 2001;18(11):797-806.

Shi X, Dalal NS, Jain AC. Antioxidant behaviour of caffeine: efficient scavenging of hydroxyl radicals. Food Chem Toxicol. $1991 ;(1): 1-6$.

Singh K, Singh S, Singhal NK, Sharma A, Parmar D, Singh MP. Nicotine- and caffeine-mediated changes in gene expression patterns of MPTP-lesioned mouse striatum: Implications in neuroprotection mechanism. Chem Biol Interact. 2010;29;185(2):81-93.

Smith A. Effects of caffeine on human behavior. Food Chem Toxicol. 2002;40(9):1243-55.

Sonsalla PK, Wong LY, Harris SL, Richardson JR, Khobahy I, Li W, Gadad BS, German DC. Delayed caffeine treatment prevents nigral dopamine neuron loss in a progressive rat model of Parkinson's disease. Exp Neurol. 2012;234(2):482-7.

Stokes AH, Freeman WM, Mitchell SG, Burnette TA, Hellmann GM, Vrana KE. Induction of GADD45 and GADD153 in neuroblastoma cells by dopamine-induced toxicity. Neurotoxicology. 2002;23(6):675-84.

Taupin P, Zini S, Cesselin F, Ben-Ari Y, Roisin MP. Subcellular fractionation on Percoll gradient of mossy fiber synaptosomes: morphological and biochemical characterization in control and degranulated rat hippocampus. J Neurochem. 1994;62(4):158695.

Tieu K. A guide to neurotoxic animal models of Parkinson's disease. Cold Spring Harb Perspect Med. 2011;1(1):a009316.

Timbrell J. Mechanism and response in cellular injury. In: Principles of Biochemical Toxicology. 3rd ed. London: Taylor \& Francis; 2003

Timson J. The effect of caffeine on the mitosis of human lymphocytes in culture. Br J Pharmacol. 1970;38(4):731-734.

Received for publication on $10^{\text {th }}$ April 2018 Accepted for publication on $14^{\text {th }}$ August 2018 